\title{
Single-exciton spectroscopy of semimagnetic quantum dots
}

\author{
J. Fernández-Rossier \\ Instituto Universitario de Materiales de Alicante, Departamento de Física Aplicada, Universidad de Alicante, \\ San Vicente del Raspeig, 03690 Spain
}

(Received 1 November 2005; published 3 January 2006)

\begin{abstract}
A photoexcited II-VI semiconductor quantum dots doped with a few Mn spins is considered. The effects of spin-exciton interactions and the resulting multispin correlations on the photoluminescence are calculated by numerical diagonalization of the Hamiltonian, including exchange interaction between electrons, holes, and Mn spins, as well as spin-orbit interaction. The results provide a unified description of recent experiments on the photoluminesnce of dots with one and many $\mathrm{Mn}$ atoms as well as optically induced ferromagnetism in semimagnetic quantum dots.
\end{abstract}

DOI: 10.1103/PhysRevB.73.045301

PACS number(s): 78.67.Hc, 75.75.+a, 78.55.Et

\section{INTRODUCTION}

Control of the wave function of spins embedded in semiconducting materials is in the road-map of the quantum hardware development. ${ }^{1}$ Optical excitation and probing of a single-exciton confined in a nanometric region permits us to manipulate the quantum state of the exciton spin, ${ }^{2,3}$ or the spin degrees of freedom coupled to the excitons such as nuclei, ${ }^{4}$ donor electrons, ${ }^{4-6}$ or $\mathrm{Mn}$ ions. ${ }^{5,7-14}$ Recent reports $^{7-9}$ of optical detections of the photoluminescence (PL) of a single quantum dot (QD) of the II-VI semiconductors doped with a single $\mathrm{Mn}$ atom showed evidence of a one on one correspondence between the energy of the emitted photons and the quantum state of the Mn spin after photon emission. Substitutional Mn in (II,Mn) VI semiconductors is a neutral impurity ${ }^{15}$ with five electrons in the open $d$ shell that behave like a spin $S=\frac{5}{2}$. Therefore, the experiment of Ref. 7 is a proof of principle of the optical manipulation and detection of the quantum state of a single spin $S=\frac{5}{2}$.

Several other groups have reported the fabrication and optical spectroscopy of a single QD of II-VI semiconductors doped with tens of Mn atoms. ${ }^{11-14,17}$ The PL spectrum of one of these semimagnetic QDs is broader than that of a pure $\operatorname{dot}^{7,12}$ and it shows strong sensitivity to the application of magnetic fields, ${ }^{12,13}$ as a result of the exchange interaction of conduction band (CB) electrons and valence band (VB) holes with the Mn spins. Here, a quantum theory of the single-exciton spectroscopy of a QD with a few Mn spins is presented. The goal is to provide a unified description of the PL spectra of QD with one ${ }^{7-9}$ and many Mn atoms, with emphasis on how to extract information about the quantum state of the Mn spins from the PL spectra. With that aim, the standard Hamiltonian for semimagnetic QD is solved exactly for $N_{M n}=1,2,3$, and $4 \mathrm{Mn}$ ions both when one exciton is present (excited state manifold, XMS) and absent (ground state manifold, GSM). The optical transition rates between the GSM and XSM are calculated and PL spectra are obtained both in the Faraday and Voigt configurations for a range of situations. Our results agree very well with the PL experiments with dots with one Mn (Ref. 7-10) and many Mn atoms. ${ }^{12,13}$ Previous theoretical work reporting exact diagonalizations ${ }^{19,20}$ has addressed single Mn dots that result in PL spectra different from those obtained here and reported experimentally, ${ }^{7,8}$ most likely due to the different symmetry of the single-hole wave functions.

The rest of the paper is organized as follows. In Sec. II we present the theoretical framework used to calculate the PL spectra of a diluted magnetic semiconductor QD. We present the second quantization Hamiltonian describing the confined electrons and holes exchanged coupled to each other and to the $\mathrm{Mn}$ ions as well as the single-particle basis for electrons and holes. The latter are obtained in the framewok of the effective mass approximation for the QD states. The most relevant properties of the many-body wave functions for the exciton coupled to the Mn spins are described later. The final ingredient is the calculation of the optical transtion rates between different many-body states. In Secs. III and IV we present the PL spectra for dots with one and more than one $\mathrm{Mn}$ atoms, respectively. In Sec. V the results are discussed together with the limitations and aproximations of the model and the conclusions of the paper.

\section{FORMALISM}

\section{A. Hamiltonian}

We consider quantum dots with a typical size of $10 \mathrm{~nm}$ and several thousand atoms, beyond reach of present-day $a b$ initio calculations. ${ }^{16}$ This justifies the use of the standard model Hamiltonian ${ }^{15,18-22}$ for diluted magnetic semiconductors that describes $\mathrm{CB}$ electrons and VB holes interacting with localized Mn spins $\left(\vec{M}_{I}\right)$ via a local exchange interaction and coupling to an external magnetic field, $\vec{B}$. The Mn spins interact also with each other via short-range superexchange coupling. This Hamiltonian has been succesfully used to describe bulk, ${ }^{15,23,24}$ two-dimensional, ${ }^{25}$ and zero-dimensional QD. ${ }^{18-22}$ In general, the Hamiltonian cannot be solved exactly and in most instances the mean field or some other approximations are used. Only in the limit of a few Mn atoms and a few electron and hole states considered in this paper is it possible to diagonalize the Hamiltonian numerically without approximations. Such a situation is relevant to understand the experimental research of QDs doped with a few $\mathrm{Mn}$ atoms. ${ }^{7-10}$ 
Both $\mathrm{CB}$ electrons and VB holes are confined in all the three spacial directions resulting in a discrete single particle spectrum. The Hamiltonian reads $\mathcal{H}=\mathcal{H}_{0}+\mathcal{H}_{1}+\mathcal{H}_{\text {eh }}$, where

$$
\mathcal{H}_{0}=-g \mu_{B} \vec{B} \sum_{I} \vec{M}_{I}+\sum_{I, J} J_{I J}^{\mathrm{AF}} \vec{M}_{I} \cdot \vec{M}_{J}
$$

and

$$
\begin{aligned}
\mathcal{H}_{1}= & \sum_{n, \sigma, \sigma^{\prime}}\left(\epsilon_{n}^{e} \delta_{\sigma, \sigma^{\prime}}-g_{e} \mu_{B} \vec{B} \cdot \frac{\vec{\tau}_{\sigma, \sigma^{\prime}}}{2}\right) c_{n, \sigma^{\dagger}}^{\dagger} c_{n, \sigma^{\prime}}+\sum_{\nu} \epsilon_{\nu}^{h}(\vec{B}) d_{\nu}^{\dagger} d_{\nu} \\
& +\sum_{I} \vec{M}_{I} \cdot\left[J_{e} \vec{S}_{e}\left(\vec{r}_{I}\right)+J_{h} \vec{S}_{h}\left(\vec{r}_{I}\right)\right] .
\end{aligned}
$$

For the low Mn concentration dots considered here the shortrange antiferromagnetic superexchange is not effective, ${ }^{21}$ although it has been included in the calculations. Therefore, in the absence of electron-hole (eh) pairs, the $\vec{M}_{I}$ interact only with $\vec{B}$. The first term in $\mathcal{H}_{1}$ describes the $\mathrm{CB}$ electrons confined in the QD orbital levels $\psi_{n}(\vec{r})$ and coupled to the external magnetic field. The Pauli matrices are denoted by $\vec{\tau}_{\sigma, \sigma^{\prime}}$ and $c_{n, \sigma}^{\dagger}$ stands for the $\mathrm{CB}$ electron creation operator. The second term describes the VB holes confined in the QD, including the spin-orbit interaction; ${ }^{26,27}$ the operator $d_{\nu}^{\dagger}$ creates a VB hole in the spin-orbital $\phi_{\nu}(\vec{r})$. The third and fourth term describes the exchange between $\mathrm{Mn}$ and $\mathrm{CB}$ electrons and VB holes, respectively. Both the CB-Mn and the VB-Mn exchange are local and couple the carrier spin density at the Mn location $\vec{S}_{e, h}\left(\vec{r}_{I}\right)$ to $\vec{M}_{I}$. The short-range eh exchange is described by $\mathcal{H}_{e h}=-J_{e h} \vec{S}_{e} \cdot \vec{S}_{h}$. Direct $e h$ Coulomb interaction and orbital magnetism are not included since they have a small effect due to the large single-particle energy spacing of the dots considered here. ${ }^{21}$

\section{B. Single-particle states}

We consider dots whose single-particle energy spacing is much larger than all the other intraband energy scales of the Hamiltonian. The shape of the dot determines the degree of light hole $(\mathrm{LH})$ heavy hole $(\mathrm{HH})$ mixing of the VB singleparticle states which, in turn, determines the relative importance of the Ising and spin flip part of the hole-Mn and holeelectron exchange interactions. A hard wall cubic potential with dimensions $L_{x}, L_{y}, L_{z}$ conveniently describes the interplay between the shape of the dot and the single-hole states, ${ }^{26}$ although real dots have more complicated geometries. We always take $L_{z}<L_{x}, L_{y}$ and we consider light propagation along the $z$ axis. The CB electron envelope functions are given by Ref. $21 \quad \psi_{\vec{n}}$ $\equiv \sqrt{8 / V} \sin \left(k_{x} x\right) \sin \left(k_{y} y\right) \sin \left(k_{z} z\right)$ where $k_{i} \equiv \pi n_{i} / L_{i}, i=x, y, z$, $V=L_{x} L_{y} L_{z}$, and $n_{i}$ are positive integers. The description of the confined VB single-particle states follows that of Ref. 26: a zero-dimensional version of the six-band Kohn-Luttinger Hamiltonian. ${ }^{27}$ The envelope states for the single-particle lowest energy doublet are $\psi_{0}(\vec{r}) \equiv \psi_{1,1,1}(\vec{r})$, both for VB holes and $\mathrm{CB}$ electrons. The wave functions of the $\mathrm{VB}$ lowest energy doublet are $|+\rangle=c_{1}\left|\frac{3}{2},+3 / 2\right\rangle+c_{2}\left|\frac{3}{2},-1 / 2\right\rangle$ and $|-\rangle=\mathcal{K} \mid$ $+\rangle$, where $\mathcal{K}$ is the time reversal operation and $|J, M\rangle$ are the standard ${ }^{27} \mathrm{LH}$ and $\mathrm{HH}$ states at the $\Gamma_{8}$ point. The weight of the split-off holes in the $| \pm\rangle$ is negligible. It would be straightforward to include the strain terms ${ }^{24}$ in this approach. Although strain is most likely relevant and changes the LH-HH splitting, it has not been included to minimize the number of parameters in the Hamiltonian.

If $L_{x}=L_{y} \mathrm{LH}-\mathrm{HH}$ mixing vanishes $\left(c_{2}=0\right)$ and so do the matrix elements $\left\langle \pm\left|S_{h}^{x, y}\right| \mp\right\rangle$, resulting in a pure Ising $(z z)$ hole-Mn and eh exchange coupling. For $0<\left|L_{x}-L_{y}\right|<L_{z}$ there is some HH-LH mixing $\left(0<\left|c_{2}\right|<\left|c_{1}\right|\right)$ that, on top of the dominant Ising coupling, permits some spin flip $(x x, y y)$ hole-Mn and $e h$ interaction. In contrast with the VB holes, the $\mathrm{CB}$ electron-Mn interaction is perfectly isotropic. ${ }^{21}$ The results shown here belong to four different dots with $\left(L_{x}, L_{y}, L_{z}\right)(7,7,4) \mathrm{nm}(\operatorname{dot} \# 1),(15,15,3) \mathrm{nm}(\operatorname{dot} \# 2)$, $(7,5,2) \mathrm{nm}(\operatorname{dot} \# 3)$, and 7,3.7,2) (dot \#4). The strength of the exchange coupling between the carriers and the Mn spins is $j_{e, h}(I) \equiv J_{e, h}\left|\psi_{0}\left(\vec{r}_{I}\right)\right|^{2}$. For a given dot the mean value of the exchange coupling is $\left\langle j_{e, h}\right\rangle=J_{e, h} / V$ and the maximum value is $8\left\langle j_{e, h}\right\rangle$. For CdTe we have ${ }^{15} J_{h}=+60 \mathrm{meV} \mathrm{nm}^{3}$ and $J_{e}=$ $-15 \mathrm{meV} \mathrm{nm}^{3}$. We take $g_{e}=-1.5$ and $g_{h}=-0.1 .^{7}$

\section{Many-body states}

The many-body states are classified according to the number of excitons $\left(N_{X}=0\right.$ for the GSM and $N_{X}=1$ for the XSM) and to the number of Mn ions, $N_{M n}$. The number of states in the GSM of a given QD is (6) ${ }^{N_{M n}}$. If only the lowest energy doublet of both the CB and VB states are kept, there are four possible exciton states, labeled by $| \pm 1\rangle_{X}$ and $| \pm 2\rangle_{X}$ following their total angular momentum, and the number of states in the XSM is $4 \times(6)^{N_{M n}}$. The many-body states are found by the standard numerical diagonalization of the Schrodinger equations $\mathcal{H}_{0}|G\rangle=E_{G}|G\rangle$ and $\left(\mathcal{H}_{0}+\mathcal{H}_{1}\right)|X\rangle=E_{X}|X\rangle$. The GSM states only feature $\mathrm{Mn}$ spin coordinates whereas the XSM states include Mn spins, as well as $1 \mathrm{CB}$ electron and $1 \mathrm{VB}$ hole that occupy linear combinations of the single-particle states described above.

In the absence of the magnetic field and superexchange all the GSM states are degenerate. In contrast, the Ising hole-Mn coupling of dot \#1 splits the 24 levels in 6 quartets, corresponding to the six possible relative orientations between the $\mathrm{Mn}$ and the hole spin along the $z$ direction. Both electron-Mn and electron-hole exchange coupling further split the 6 quartets into 12 doublets. Depending on the relative size of $J_{e h}$ and $J_{e}$ the lowest energy state of the XSM is either $| \pm 1\rangle_{X}$ $\otimes\left|\mp \frac{5}{2}\right\rangle$ or $| \pm 2\rangle_{X} \otimes\left|\mp \frac{5}{2}\right\rangle$. The diagonalizations show that the lowest energy states of the XSM of dots with $N_{M}=2,3,4$ describe fully polarized Mn spins along $L_{z}$. Hence, the exact many-spin wave functions of the Mn coupled to zerodimensional exciton feature ferromagnetic correlations, very much like bulk carrier mediated ferromagnetic order ${ }^{23,25}$ and in agreement with experimental observations in photoexcited QD. ${ }^{14}$

\section{Optical transitions}

Transitions from the XSM to the GSM are possible via spontaneous emission of a photon with the adequate energy 
$\hbar \omega$ and polarization. We consider circularly polarized photons. The rate of spontaneous emission of a photon of energy $\hbar \omega=E_{X}-E_{G}$ from the state $|X\rangle$ to the state $|G\rangle$ is given by the Weisskopf-Wigner expression $\Gamma_{G X}=4 \omega^{3} / 4 \pi \epsilon \hbar c^{3}\left|\left\langle G\left|\mathcal{P}^{ \pm}\right| X\right\rangle\right|^{2}$, where

$$
\mathcal{P}^{ \pm}=\sum_{\nu, n, \sigma}\langle\nu|e(x \pm i y)| n \sigma\rangle d_{\nu}^{\dagger} c_{n, \sigma}^{\dagger}+\text { H.c. }
$$

is the second quantization representation of the interband electric dipole operator that yields the standard optical selection rules. Standard optical selection rules forbid photon emission from $| \pm 2\rangle_{X}$ states. Since the electric dipole operator does not affect the Mn $d$ electrons, the Mn spin part of the collective wave function does not change during the recombination process. As a result, most of the GSM to XSM transitions that are allowed by the standard optical selection rule but are forbidden due to the orthogonality of the spin part of GSM and the XSM states. This optical spin blockade, reminiscent of the spin blockade well characterized in the single electron transport, ${ }^{28}$ implies a huge limitation to the otherwise large set of XSM to GSM transitions. The PL spectrum is calculated by statistical weighting of the initial states $|X\rangle$ :

$$
\mathcal{I}_{P L}(\omega)=\sum_{X, G} P(X) \Gamma_{G X} \delta\left(\hbar \omega-\left(E_{X}-E_{G}\right)\right),
$$

where $P(X)$ is the occupation probability of the XSM state $|X\rangle$. In general, the calculation of $P(X)$ would involve the determination of the nonequilibrium steady state density matrix. ${ }^{18,19}$ The experimental results of Ref. 7 support approximating $P(X)$ as a thermal distribution function with an effective temperature.

\section{DOTS WITH ONE MAGNETIC ION}

\section{A. Results without eh exchange}

We now show PL spectra of QD doped with a single Mn atom, calculated following the method described above. In order to establish the influence of the different exchange couplings in the PL spectra of dots with $1 \mathrm{Mn}$, we first take $J_{e h}=0$ (Fig. 1) and then we study the effect of $e h$ exchange (Fig. 2). The spectral lines are broadened using Gaussians with a FWHM of either $\gamma=50 \mu \mathrm{eV}$ or $\gamma=400 \mu \mathrm{eV}$, adapted to the experimental photodetector resolution of Refs. 7 and 12, respectively. Single spin optical spectroscopy calculations are shown in Fig. 1. The zero field PL for QD \#1 with a single $\mathrm{Mn}$ impurity and two effective temperatures, 0.5 and $2 \mathrm{meV}$ (6 and $24 \mathrm{~K}$ ) is shown in Fig. 1(a). The model accounts quantitatively for the experimental observations: ${ }^{7}$ only 6 lines are clearly seen, in spite of the fact that the XSM has 12 nondegenerate lines that occupy a spectral range of $1 \mathrm{meV}$. The number of the lines differ from those of previous theory work ${ }^{19,20}$ due to the different symmetry of the heavy hole wave function considered here. The six lines undergo a polarization dependent splitting [Fig. 1(b), lower spectrum] upon application of a magnetic field in the Faraday configuration, $\vec{B}=\left(0,0, B_{z}\right)$, in good agreement with the experiments. ${ }^{7,10}$
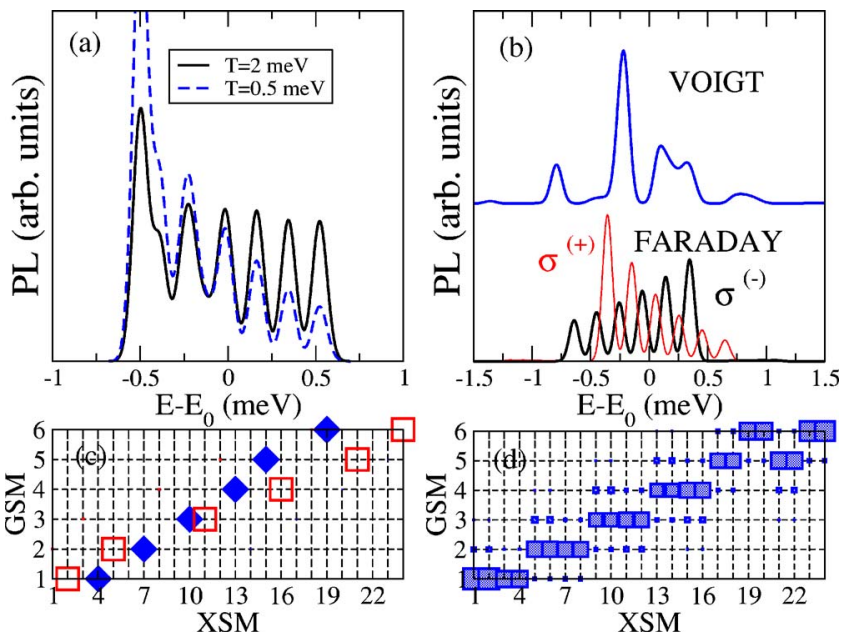

FIG. 1. (Color online) (a) PL for dot \#1 with $1 \mathrm{Mn}$ atom and two values of T. (b) Magneto PL in the Faraday $[(0,0,5 T)]$ and Voigt $[(5 T, 0,0)]$ configurations. (c) and (d) $\Gamma_{G X}$ for the Faraday (c) and Voigt (d) cases.

The detection of a photon with a given polarization and energy in one of these six peaks yields information about the final state of the GSM in the optical transition. That information is given, for the Faraday spectrum of Fig. 1(b), in Fig. 1(c). There the size of the symbols is proportional to $\Gamma_{G X}$ for $\sigma_{-}$(open squares circles) and $\sigma_{+}$(solid diamonds). It is seen how each member of the XSM is coupled via a given circularly polarized photon with, at most, one member of the GSM. This fact permits us to map the photon state (energy and polarization) to the final Mn state and is one of the main results of this paper. In particular, going from low to high energy the six peaks from the PL in the Faraday case correspond to final Mn states going from $S_{z}=+\frac{5}{2}$ to $S_{z}=-\frac{5}{2}$. The zero field case has the same one-on-one correspondence.

The calculated PL in the Voigt configuration, $\vec{B}$ $=\left(B_{x}, 0,0\right)$, is remarkably different. The shape of the PL is modified drastically, and the spectrum is much wider. The GSM states are the eigenstates of the $S_{X}$ spin operator, whereas the XSM states have strong overlaps with the eigen-

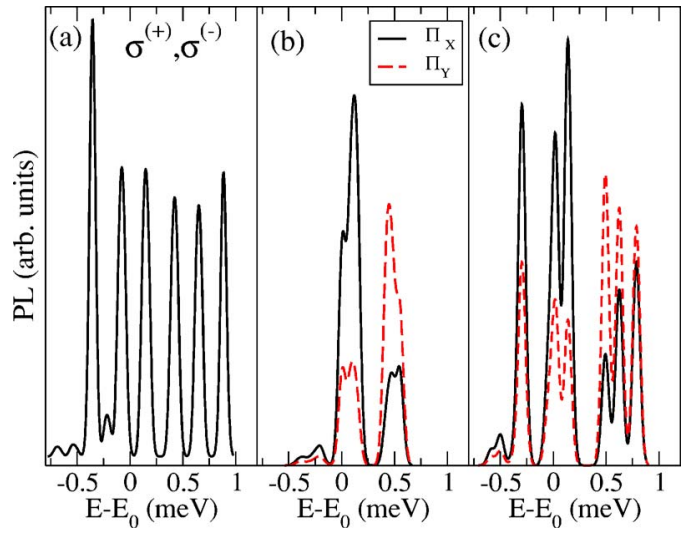

FIG. 2. (Color online) Influence of the LL-HH mixing and the eh exchange on the PL of single Mn PL for dots \#3 (a) and \#4 (b and (c). 
states of the $S_{z}$ operator, because the spin of the hole is pinned in that direction and the hole-Mn Ising coupling is dominant. The matrix $\langle G|\mathcal{P}| X\rangle$ is now proportional to $\left\langle S_{x} \mid S_{z}\right\rangle$ making the spin blockade much less efficient, as shown in Fig. 1(d). This is seen in Fig. 1(d) and accounts for the PL shape reported in Ref. 10

Since the optical absorption selection rules are also given by $\Gamma_{G X}$, Fig. 1(d) also tells us something about the ground state spin coherence induced by two-photon processes. ${ }^{5,6} \mathrm{It}$ is seen that the GSM ground state $\left|S_{x}=+\frac{5}{2}\right\rangle$ is coupled to the first eight states in the XSM via a one-photon absorption. In turn, these are coupled to states $\left|S_{x}=+\frac{5}{2}\right\rangle,\left|S_{x}=+\frac{3}{2}\right\rangle, \mid S_{x}=$ $\left.+\frac{1}{2}\right\rangle$ and $\left|S_{x}=-\frac{1}{2}\right\rangle$ via one-photon emission. These two-photon processes can produce spin coherence between states with different energy results in a long lived magnetization precession. ${ }^{5,29}$

\section{B. Results with $e h$ exchange}

We now consider the effect of the short-range eh exchange interaction, absent in the results of Fig. 1. The symmetry properties of the short-range $e h$ exchange are identical to those of the $\mathrm{Mn}-h$ coupling, since both are related to the same hole-spin operator. The Ising part of the eh interaction splits bright and dark excitons, whereas the spin-flip part of the $e h$ interaction, which is only possible if there is LH-HH mixing, mixes +1 and -1 excitons resulting into linearly polarized spectra. ${ }^{2}$ Long-range exchange $e h$ exchange also mixes +1 and -1 states, ${ }^{30}$ but is not included in the calculation in order to keep the number of parameters in the Hamiltonian to a minimum. In the presence of $\mathrm{Mn}$, this mixing competes with the Ising hole-Mn coupling. The combined action of electron-Mn exchange and the transverse hole-Mn and $e h$ interactions mix somewhat bright and dark excitons. In Figs. 2(a)-2(c) we show the PL spectra corresponding to dot \#3 [(2a)] and dot \#4 [(2b and 2c)], all of them with a single $\mathrm{Mn}$ atom and $J_{e h}=1 \mathrm{meV}$. Dot \#3 features some LH-HH mixing. Ising $e h$ interaction redshifts the dark excitons, resulting in the appearance of three low energy peaks (compared with Fig. 1). The middle PL corresponds to a Mn weakly coupled $\left(j_{h}=0.16 \mathrm{meV}, j_{e}=0.25 j_{h}\right)$ to the exciton and a strong LH-HH mixing. The PL is linearly polarized and the peak structure of Fig. 1(a) is not resolved. The lower panel corresponds to the same dot with a strongly coupled Mn $\left(j_{h}=0.31 \mathrm{meV}\right)$. There the six peak structure is recovered, but the PL is linearly polarized, mostly in the central peaks for which the hole-Mn Ising coupling is smaller. This whole picture is consistent and qualitatively identical to recently published experiments. ${ }^{9}$ The fact that the model is able to account for the fine structure of a variety (Refs. 7 and 9) of spectra implies that it captures correctly the symmetry properties of all the exchange interactions relevant in the system.

\section{DOTS WITH MORE THAN ONE MAGNETIC ION}

Here we address the central theme of this paper: how the PL spectra of the dot evolve as the number of Mn atoms increases. In that regard, we start considering the case of two impurities whose relative coupling to the fermions is defined
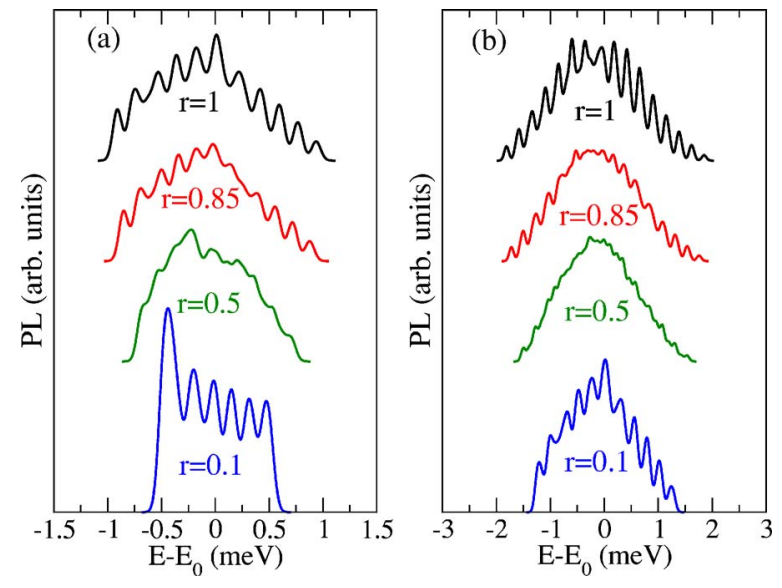

FIG. 3. (Color online) (a) Zero field PL spectra for dot \#2 with two Mn impurities as a function of the relative Mn-exciton couplings. (b) Zero field PL spectra for dot \#2 with three Mn impurities, two of them equally coupled to the exciton, as a function of the relative $\mathrm{Mn}$-exciton coupling.

by $r=\left|\psi_{0}\left(\vec{r}_{2}\right)\right|^{2} /\left|\psi_{0}\left(\vec{r}_{1}\right)\right|$. We consider dot \#2. In Fig. 3(a) we explore the effect of the relative Mn-hole coupling on the PL; the position of Mn 1 is fixed so that its coupling to the hole is $j_{h}=0.29 \mathrm{meV}$, and $\vec{r}_{2}$ is varied so that $r$ goes from 1 to 0.1 (from top to bottom). The symmetric case $r=1$ shows a clean PL spectrum with 11 lines corresponding to quantum states with the $2 S+1$ possible orientations of the collective $S_{Z}$ of the two Mn atoms $(S=5)$. These 11 peaks are still seen for $r=0.85$ and, with some numerical analysis of the data, even for $r=0.5$. When $r=0.1$ the PL spectrum looks like that of a dot with only one $\mathrm{Mn}$ atom. This indicates that weakly coupled Mn spins are undetectable by optical means. The same evolution is shown in Fig. 3(b) for the case of three impurities with $j_{h}(1)=j_{h}(2)=0.38 \mathrm{meV}$ and $r=j_{h}(3) / j_{h}(1)$. In this case the PL spectrum features 16 peaks for $r=1$ corresponding to the projections of $S_{z}$ with $S=\frac{15}{2}$, and crosses over to 11 peaks for $r=0.1$.

The results of Fig. 3 suggest that a few Mn atoms, strongly coupled to the exciton, could be responsible for the intinsic broad linewidth of the PL signal observed in a single QD doped with many Mn atoms. In Ref. 12 the PL spectrum of a single semimagnetic QD show a zero field $\sigma^{+}$line with a full width at half maximum (FWHM) of $\simeq 5 \mathrm{meV}$ that redshifts and narrows upon application of a magnetic field in the Faraday configuration. ${ }^{11-13}$ This has motivated statistical interpretation of the PL spectrum of single semimagnetic $\mathrm{QD},{ }^{12,13}$ linking the PL linewidth to the magnetic statistical fluctuations. In such an approach the PL FWHM has been shown to scale with $\sqrt{k_{B} T / V}$. On the other side, it is apparent that the PL spectrum of a single Mn impurity ${ }^{7}$ lies in a window of order $\Delta_{I}=\frac{5}{2}\left[\left|j_{h}(I)\right|+\left|j_{e}(I)\right|\right]$, which scales like $V^{-1}$. The PL linewidth of Fig. 2 also scales with $\Sigma_{I} \Delta_{I} \propto V^{-1}$.

We have explored whether the quantum approach with $N_{M n}=3$ and 4 can model PL spectra like those of Refs. 11-13. The justification lies in the distribution of couplings $j_{h}(I)$, shown in Fig. 4(a) for $\mathrm{Cd}_{0.99} \mathrm{Mn}_{0.01} \mathrm{Te}$ (dot \#1) obtained by random generation of 100 realizations of the Mn disorder. Such a dot has $N_{M n}=38$ and $\left\langle j_{h}\right\rangle=0.3 \mathrm{meV}$. However, $65 \%$ 


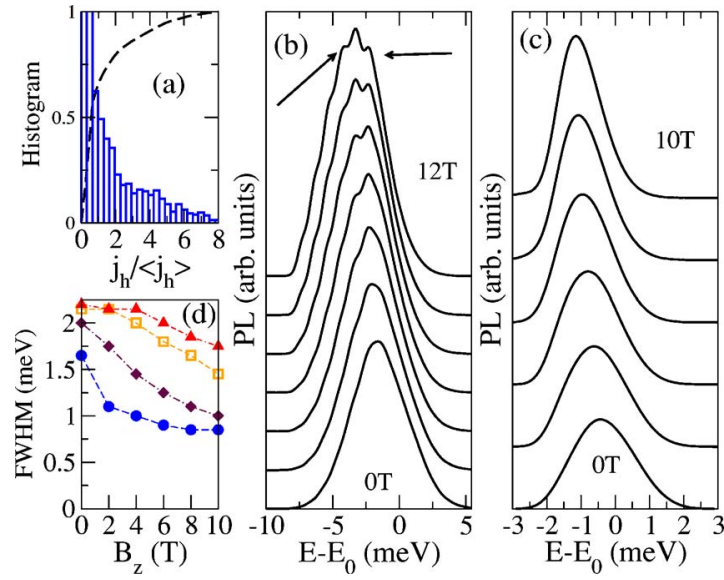

FIG. 4. (Color online) (a) Normalized histogram of Mn-hole coupling. The dashed line is the integrated histogram. (b) PL spectrum for four Mn impurities (see text) for several values of $B_{z}$. (c) Idem for three Mn impurities. (d) FWHM for as a function of $B_{z}$ and $T=3,2,1$, and $0.5 \mathrm{meV}$ (top to bottom).

of the Mn have $j_{h}<\left\langle j_{h}\right\rangle$ and, in average, the four Mn with the largest coupling have $j_{h}>3\left\langle j_{h}\right\rangle$. We calculate the PL taking the four Mn atoms with the largest overlap to the carrier wave function, for a randomly selected Mn disorder realization. Their $j_{h}$ are 1.69, 1.66, 1.1, and $0.45 \mathrm{meV}$. In Fig. 4(b) we show the corresponding $\sigma^{+}$PL spectrum for values of the magnetic field going from zero to $12 \mathrm{~T}$ in the Faraday configuration. We take $\gamma=0.4 \mathrm{meV}$ and $T=3 \mathrm{meV}$. The bell shape of the calculated spectra comes from the many-body density of states and is not due to the broadening $\gamma$. As in the experiments, the $\sigma^{+}$PL redshifts as $B_{z}$ goes up. The featureless zero field PL spectrum (with a FWHM of $5 \mathrm{meV}$ ) develops, as $B_{z}$ increases narrow structures, as reported experimentally. ${ }^{13}$ The narrowing is also seen in Fig. 4(c) that shows the PL of dot \#1 with three Mn atoms, with values of $j_{h}$ closer to the average $(0.39,0.40$, and $0.44 \mathrm{meV})$, with $T$ $=2 \mathrm{meV}$ and $\gamma=0.4 \mathrm{meV}$. In Fig. 4(d) we show how the corresponding FWHM decreases both upon cooling and upon increasing $B_{z}$. The former is due to the reduction of the spectral range of the occupied states in the XSM, resulting as well in a redshift that has been observed experimentally. ${ }^{12}$ The magnetic field produces a similar effect: the $|X\rangle$ states that emit $\sigma^{+}\left(\sigma^{-}\right)$photons shift towards the red (blue) and become relatively more (less) occupied.

\section{DISCUSSION, APPROXIMATIONS, AND CONCLUSIONS}

\section{A. Discussion}

So far we have provided a theoretical framework to understand the PL spectra of single QDs of II-VI semiconductors doped with a few Mn atoms. The theoretical framework involves the standard diluted magnetic semiconductor Hamiltonian for electrons and holes exchanged-coupled to the Mn ions, with the particular feature that electrons and holes are confined by the dot potential. The size of the dot controls the strength of the carrier-Mn coupling and the single-particle level spacing whereas the shape of the dot affects the heavy hole-light hole coupling which in turn determines the symmetry of the exchange interaction of the hole with the Mn ions and with the electron. For a sufficiently small number of $\mathrm{Mn}$ atoms (four in this work) the size of the Hilbert space of confined fermions and Mn spins permits the exact numerical diagonalization of the Hamiltonian. The PL spectra are obtained from the exact eigenstates of the Hamiltonian using the standard equation (4).

Broadly speaking, the single dot PL spectra available so far have either well defined peaks that can be attributed to exciton transitions coupled to a single $\mathrm{Mn}$ atom ${ }^{7-10}$ or broad PL spectra that have been interpreted in terms of excitons coupled to many Mn atoms. ${ }^{11-13}$ It is our contention that the two kind of PL spectra can be described using the microscopic approach described above. In the case of the "single Mn dots" the model permits to fit a variety of PL spectra of different dots (Figs. 1 and 2). This procedure yields information about the size of the different exchange coupling constants in the Hamiltonian for each dot. On the other side, the model gives a more fundammental information: in the case of PL spectra with six resolved major peaks, there is a one on one correspondence between the quantum state of the $\mathrm{Mn}$ spin after the photon emission and the energy and polarization of the detected photon. This correspondence permits the detection of the quantum state of a single $\mathrm{Mn}$ spin conditioned to the detection of the photon state (energy and polarization). The detection of the quantum state of an isolated quantum system is one of the requirements in the implementation of a quantum computation making the Mn-doped QD interesting systems in that regard.

The mapping of the Mn state onto the photon energy is made possible by the strong anisotropy of the hole-Mn coupling of certain dots. The most favorable case is a dot in which the single-hole ground state is purely heavy hole (no mixing with the light hole states). In that case the Mn-hole coupling is purely Ising so that the 12 possible hole-Mn states are resolved in six doublets with well defined $\mathrm{Mn} M_{z}$. This anisotropy comes ultimately from the interplay between spin-orbit interaction of the host material, which is responsible for most of the bulk magnetic anisotropies, ${ }^{23,24}$ and the breaking of the crystal translational invariance, which causes new effects like tunneling anisotropic magnetoresistance in transport through tunnel barriers ${ }^{31}$ of diluted ferromagnetic semiconductors.

The PL spectra of QDs where the exciton is coupled to more than one Mn are substantially more complicated. In the rare event that the exciton is coupled with equal or similar strength to all of the Mn the many-body spectrum will have additional degeneracies related to such a symmetry. In such situations the number of resolved peaks would be observed in the PL, as in Fig. 3. In most instances the exciton will be more coupled to some Mn ions and the resulting PL spectra will be featureless. The width of a typical PL spectra in our model comes from the fact that the states of the XSM are distributed in a range of energies. Such a width is an intrinsic property of the involved structure of the many-body spectrum of the exciton-Mn problem. Application of the magnetic field spin splits the many-body states, increasing the relative occupation of the lower energy states and decreasing the width of the PL spectra thereby. The main conclusion drawn 
from the simulations shown in Fig. 3 is that the PL spectra of an exciton coupled to only three or four $\mathrm{Mn}$ atoms are already as dense and broad as those observed experimentally.

\section{B. Limitations of the theoretical framework}

Our theoretical framework accounts fairly well for a variety of experimental results but it contains some approximations and limitations. Since the model-Hamiltonian is diagonalized exactly the approximations occur in the derivation of the model. The $e h$ direct Coulomb interaction, long-range $e h$ exchange, orbital mangnetism of both electrons and holes, and effect of the strain on the hole spectrum have been not included in the Hamiltonian. The use of an effective mass description of the single-particle states, as well as the choice of a hard-wall quantum dot model are also approximations.

The direct Coulomb interaction shifts the optical absorption energy and mixes the different single-particle states. The former is irrelevant since there was no attempt to predict the absolute value of the optical band gap. The latter is only important if the single-particle level spacing is comparable to the exciton binding energy. The same applies to the effect of the magnetic field on the orbital part of the wave function: it matters if the cyclotron energy is comparable to the singleparticle spacing. The modification of the wave function would yield a change in the exchange integrals as well as in the optical selection rules as a function of the magnetic field. The calculation of the single-particle states including the effect of the magnetic field on the orbits is out of the scope of this paper. In contrast, the effect of the magnetic field on the spin is fully taken into account and is relevant at arbitrary small fields.

Further theory work would be necessary to study dots with smaller energy spacing for which Coulombic and dia- magnetic effects are more relevant. The effect of long-range exchange $e h$ exchange interactions and strain need also further work. The results presented here can be considered a first approximation in that direction.

\section{Conclusions}

In conclusion, the problem of a few Mn atoms interacting with a single exciton in a quantum dot is solved via numerical diagonalization of the Hamiltonian including electron$\mathrm{Mn}$, hole-Mn, electron-hole exchange as well as spin-orbit interactions, and the correponding PL spectra are calculated. The model accounts for a series of recent reports with the PL of CdTe dots doped with a single Mn atom and permits us to link the quantum state of one $\mathrm{Mn}$ spin to the detection of a photon with a given energy and polarization. The crucial ingredient for this one on one correspondence is the anisotropy of the hole-Mn interaction, due to strong spin-orbit interaction for the holes. The model also reproduces qualitatively the PL spectra of single dots with presumably tens of Mn atoms ${ }^{11-13}$ with just three and four Mn spins strongly coupled to the exciton and with different coupling strengths. The detailed understanding that emerges from the interplay between theory and experiments can pave the road towards control of the wavefunction of several Mn spins using laser pulses.

\section{ACKNOWLEDGMENTS}

I would like to thank L. Besombes, Y. Leger, and H. Mariette for their support. Fruitful discussions with J. J. Palacios and funding from Grant No. FIS200402356 and the Ramon y Cajal Program (MEC, Spain) are acknowledged. Funding from the Generalitat Valenciana GV05-152 is acknowledged. This work has been partly funded by the FEDER.
${ }^{1}$ B. E. Kane, Nature (London) 393, 133 (1998); D. Loss and D. P. DiVincenzo, Phys. Rev. A 57, 120 (1998).

${ }^{2}$ N. H. Bonadeo, J. Erland, D. Gammon, D. Park, D. S. Katzer, and D. G. Steel, Science 282, 1473 (1998).

${ }^{3}$ X. Li, Y. Wu, D. Steel, D. Gammon, T. H. Stievater, D. S. Katzer, D. Park, C. Piermarocchi, and L. J. Sham, Science 301, 809 (2003).

${ }^{4}$ F. Jelezko, T. Gaebel, I. Popa, M. Domhan, A. Gruber, and J. Wrachtrup, Phys. Rev. Lett. 93, 130501 (2004).

${ }^{5}$ J. Bao, A. V. Bragas, J. K. Furdyna, and R. Merlin, Nat. Mater. 2, 175 (2003).

${ }^{6}$ P. Chen, C. Piermarocchi, L. J. Sham, D. Gammon, and D. G. Steel, Phys. Rev. B 69, 075320 (2004).

${ }^{7}$ L. Besombes, Y. Leger, L. Maingault, D. Ferrand, H. Mariette, and J. Cibert, Phys. Rev. Lett. 93, 207403 (2004).

${ }^{8}$ L. Besombes, Y. Leger, L. Maingault, D. Ferrand, H. Mariette, and J. Cibert, Phys. Rev. B 71, 161307(R) (2005).

${ }^{9}$ Y. Léger, L. Besombes, L. Maingault, D. Ferrand, and H. Mariette, Phys. Rev. Lett. 95, 047403 (2005).

${ }^{10}$ Y. Léger, L. Besombes, L. Maingault, D. Ferrand, and H. Mariette (unpublished).

${ }^{11}$ A. A. Maksimov, G. Bacher, A. McDonald, V. D. Kulakovskii, A.
Forchel, C. R. Becker, G. Landwehr, and L. Molenkamp, Phys. Rev. B 62, R7767 (2000).

${ }^{12}$ G. Bacher, A. A. Maksimov, H. Schoemig, V. D. Kulakovskii, M. K. Welsch, A. Forchel, P. S. Dorozhkin, A. V. Chernenko, S. Lee, M. Dobrowolska, and J. K. Furdyna, Phys. Rev. Lett. 89, 127201 (2002).

${ }^{13}$ P. S. Dorozhkin, A. V. Chernenko, V. D. Kulakovskii, A. S. Brichkin, A. A. Maksimov, H. Schoemig, G. Bacher, A. Forchel, S. Lee, M. Dobrowolska, and J. K. Furdyna, Phys. Rev. B 68, 195313 (2003).

${ }^{14}$ S. Mackowski, T. Gurung, T. A. Nguyen, H. E. Jackson, L. M. Smith, G. Karczewski, and J. Kossut, Appl. Phys. Lett. 84, 3337 (2004).

${ }^{15}$ J. K. Furdyna, J. Appl. Phys. 64, R29 (1988).

${ }^{16}$ X. Huang, A. Makmal, J. R. Chelikowsky, and L. Kronik, Phys. Rev. Lett. 94, 236801 (2005).

${ }^{17}$ S. C. Erwin, L. Zu, M. I. Haftel, A. L. Efros, T. A. Kennedy, and D. J. Norris, Nature (London) 436, 91 (2005).

${ }^{18}$ A. I. Efros, M. Rosen, and E. I. Rashba, Phys. Rev. Lett. 87, 206601 (2001)

${ }^{19}$ A. O. Govorov, Phys. Rev. B 70, 035321 (2004); A. O. Govorov and A. V. Kalameitsev, ibid. 71, 035338 (2005). 
${ }^{20}$ A. K. Bhattacharjee and J. Pérez-Conde, Phys. Rev. B 68, 045303 (2003).

${ }^{21}$ J. Fernández-Rossier and L. Brey, Phys. Rev. Lett. 93, 117201 (2004).

${ }^{22}$ A. O. Govorov, Phys. Rev. B 72, 075359 (2005); 72, 075358 (2005); J. I. Climente, M. Korkusinski, P. Hawrylak, and J. Planelles, ibid. 71, 125321 (2005).

${ }^{23}$ T. Dietl, T. Dietl, H. Ohno, F. Matsukura, J. Cibert, and D. Ferrand, Science 287, 1019 (2000).

${ }^{24}$ M. Abolfath, T. Jungwirth, J. Brum, and A. H. MacDonald, Phys. Rev. B 63, 054418 (2001).

${ }^{25}$ J. Fernández-Rossier and L. J. Sham, Phys. Rev. B 64, 235323
(2001); 66, 073312 (2002).

${ }^{26}$ F. V. Kyrychenko and J. Kossut, Phys. Rev. B 70, 205317 (2004).

${ }^{27}$ J. M. Luttinger and W. Kohn, Phys. Rev. 97, 869 (1955).

${ }^{28}$ J. J. Palacios, L. Martin-Moreno, G. Chiappe, E. Louis, and C. Tejedor, Phys. Rev. B 50, R5760 (1994); D. Weinmann, W. Hausler, and B. Kramer, Phys. Rev. Lett. 74, 984 (1995).

${ }^{29}$ S. A. Crooker, J. J. Baumberg, F. Flack, N. Samarth, and D. D. Awschalom, Phys. Rev. Lett. 77, 2814 (1996).

${ }^{30}$ E. L. Ivchenko, Phys. Status Solidi A 165, 487 (1997).

${ }^{31}$ L. Brey, C. Tejedor, and J. Fernández-Rossier, Appl. Phys. Lett. 85, 1996 (2004) 